\title{
Long-term health-related quality of life concerns related to the COVID-19 pandemic: a call to action
}

\author{
W. Jeff Bryson ${ }^{1} \mathbb{0}$
}

Accepted: 7 October 2020 / Published online: 18 October 2020

(c) Springer Nature Switzerland AG 2020

\begin{abstract}
The COVID-19 pandemic has far-reaching implications for HRQoL into the future. This disease affects many systems of the body, as well as having an impact on the psychological status of those with the condition, their families, and health care workers. Given the breadth of conditions that may result from COVID-19, it is critical that quality of life researchers begin preparing to address the many factors that may impact HRQoL for many people worldwide.
\end{abstract}

Keywords Health-related quality of life $\cdot$ COVID-19 $\cdot$ Long-term complications $\cdot$ Health care avoidance

The novel coronavirus (COVID-19; SARS-CoV-2) pandemic has affected millions worldwide and enveloped every continent with the possible exception of Antarctica. COVID19 is highly transmissible and associated with many potential health consequences [1-4]. Scientific endeavors to better understand this virus and its complications are ongoing, but generally inadequate at this time due to the recency of the disease and the lack of large-scale and longitudinal studies. Some health consequences such as pneumonia and increased proinflammatory cytokine levels are associated with the virus or immune response [1,2]. Those who contract the virus are also at risk for developing complications such as those involving the respiratory, neurological, and renal systems [1-4]. Efforts to prevent the virus may also have a broader impact on health conditions not directly related to COVID-19. An example of this is the possible avoidance of health care due to fears of contracting COVID-19 in the process. Those in higher risk categories such as advanced age, diabetes, cardiovascular disease, kidney disease, or compromised immune systems may be appropriately fearful, but also at risk of jeopardizing their health status if critical monitoring and treatments are reduced. Any chronic illness that may arise directly from COVID-19 or from maladaptive

W. Jeff Bryson

jeff.bryson@alliancehsv.com

1 Alliance Behavioral Health, LLC, 250 Chateau Drive, Suite 145, Crestwood Professional Center, Huntsville, AL 35801, USA responses to prevent COVID-19 will have the potential to negatively affect health-related quality of life (HRQoL) [5].

Pneumonia and ground glass opacities are commonly observed in those with COVID-19 [1]. Although many will recover, there may be long-term damage and slower recovery among some survivors. This may leave those affected individuals with hypoxia, shortness of breath, and limitations in the ability to work and be active. Changes in a person's level of functioning are also expected to contribute to depression and anxiety. This may also lead to poorer HRQoL stemming from factors such as pain, fatigue, reduced ability to contribute to family resources or community responsibilities, and an altered sense of identity and purpose.

SARS-CoV-2, as with other coronaviruses, has the potential to be neuroinvasive [3]. Hypoxia is another concern that may arise with COVID-19 and has the potential to affect neurological functioning $[1,2]$. There are widespread reports of neurological sequelae and it is yet to be seen if these may become chronic in nature. Reports of dysgeusia and anosmia are commonly reported among those with COVID-19 [3]. Seizures, convulsions, ataxia, encephalitis, and demyelination are also seen in some cases [2,3]. Changes in mental status are reported and may manifest as significant neuropsychological impairments including depression, anxiety, delirium, confusion, poor attention and concentration, memory loss, and executive dysfunction $[2,3]$. There should also be concern for the potential emergence of traumatic stressrelated disorders among survivors, health care workers, and family members. Again, all of these manifestations potentially having broad-reaching negative effects on HRQoL. 
COVID-19 commonly affects the kidneys including the presence of hematuria, proteinuria, and acute kidney injury (AKI) [4]. This often resolves within a few weeks for most patients. Kidney impairments in those with COVID-19 were associated with higher mortality [4]. It is well known that those with preexisting kidney disease are also classified in the higher risk for mortality category. The overall risk for chronic kidney disease (CKD) after COVID-19 is unclear and could take years to fully establish. Those with preexisting kidney disease are also at risk if they engage in health care avoidance during the pandemic. If chronic kidney disease arises from COVID-19, this would also be expected to result in depression, pain, fatigue, and ultimately, a poorer HRQoL [5].

Knowledge about COVID-19 is still accumulating daily. The long-term effects of COVID-19 and its impact on HRQoL has not had sufficient time to fully develop and manifest in order to be effectively studied. Current efforts are underway to address the many issues related to COVID19. This includes methods of prevention, improving the speed, accuracy, and availability of testing, development of vaccines, better understanding the pathophysiology and long-term consequences that may arise, and determining treatment efficacy for a variety of pharmacological agents. Addressing core outcomes sets (COS) is essential to ensuring that as much needed information is available to those making decisions about appropriate interventions. The COMET initiative has made links available to COS studies related to COVID-19 interventions in a variety of settings and populations [6]. Researchers are encouraged to review this information.

It is time that HRQoL researchers begin to consider the role they will play in the near future. The initial step will be to devise studies to better understand the relationships of the various factors that affect those who have been infected with COVID-19 and those who are involved in the care of these individuals. Given the myriad systems that COVID19 can affect, it is essential that efforts address a variety of populations and conditions $[1-4,6]$. It is also important to consider the international implications in that different regions, groups, and cultures may experience differences in exposure, coping responses, availability of resources, and socioeconomic impacts. Addressing issues of health care disparities within nations and internationally will be needed due to the potential impact this may have on testing, treatment, vaccination studies, and access to quality health care. Future studies should also address the outcomes for interventions and how these are related to HRQoL [6]. The impact of potential health care avoidance due to fears of COVID-19 exposure should also be addressed as a factor that may later affect HRQoL.

Patient-reported outcome measures (PROMs) are widely available that would be beneficial for use now. Many of these have been well established over time, have sound psychometric properties, and are broadly applicable. PROMs could be used in a variety of combinations to target information from multiple settings, health conditions, and populations. Of course, other measures may need to be developed that address specific aspects of COVID-19. For example, the Coronavirus Anxiety Scale was recently developed as a screener for anxiety related specifically to COVID-19 [7]. Such tools may be beneficial in further assessing healthrelated anxiety and health care avoidance during the pandemic. Avoidance of health care related to COVID-19 is currently estimated at $41 \%$ among the U.S. adult population and, again, may have deleterious effects on health and HRQoL even among those without COVID-19 infections [8]. The use of currently available screeners and PROMs, as well as the potential development of new measures specific to the pandemic will be needed to ensure optimal data for further decision making about prevention, care, and utilization of resources.

COVID-19 will likely be a point of origin for broader long-term impairments in a subset of survivors, families, and health care workers that will also need to be addressed over time. Regardless of the specific nature of the longterm consequences (respiratory, neurological, renal, psychological, etc.), there will likely be deleterious effects on HRQoL. Given the large-scale impact on health in a multitude of forms, it is likely that the end result may be a sharp increase in the number of people worldwide with poorer HRQoL. Early studies should focus on identifying key factors involved in HRQoL in a variety of populations and conditions, as well as resources that may be needed to address these issues. Later studies should focus on how various interventions including vaccines, medications, resource allocations, and psychosocial interventions affect HRQoL and if these resources are being made available to the populations in need. It is imperative that researchers begin preparing to address a variety of potential etiologies and factors that may negatively impact HRQoL among survivors, families, and health care workers in the near future.

\section{Compliance with ethical standards}

Conflict of interest The author has no financial support, off-label or investigational uses, or other conflicts of interest to report.

\section{References}

1. Hosseiny, M., Kooraki, S., Gholamrezanezhad, A., Reddy, S., \& Myers, L. (2020). Radiology perspective of coronavirus disease 2019 (COVID-19): Lessons from severe acute respiratory 
syndrome and Middle East respiratory syndrome. American Journal of Roentgenology, 214(5), 1078-1082.

2. Rothan, H. A., \& Byrareddy, S. N. (2020). The epidemiology and pathogenesis of coronavirus disease (COVID-19) outbreak. Journal of Autoimmunity. https://doi.org/10.1016/j.jaut.2020.102433.

3. Baig, A. M. (2020). Neurological manifestations in COVID-19 caused by SARS-CoV-2. CNS Neuroscience \& Therapeutics, 26(5), 499.

4. Pei, G., Zhang, Z., Peng, J., Liu, L., Zhang, C., Yu, C., et al. (2020). Renal involvement and early prognosis in patients with COVID-19 pneumonia. Journal of the American Society of Nephrology, 31(6), 1157-1165.

5. Wang, H. M., Beyer, M., Gensichen, J., \& Gerlach, F. M. (2008). Health-related quality of life among general practice patients with differing chronic diseases in Germany: Cross sectional survey. BMC Public Health, 8(1), 246. https://doi. org/10.1186/1471-2458/8/246

6. Williamson, P., Gargo, L., Clarke, M., Marshall, J., Zhang, J., Qiu, R., et al. (2020). Core outcome set developers' response to COVID-19. Resource document. The COMET Initiative. Retrieved September 25, 2020, from https://www.comet-initiative .org/Studies/Details/1538.

7. Lee, S. A. (2020). Coronavirus Anxiety Scale: A brief mental health screener for COVID-19 related anxiety. Death Studies, 44(7), 393-401.

8. Czeisler, M. É., Marynak, K., Clarke, K. E., Salah, Z., Shakya, I., Thierry, J. M., et al. (2020). Delay or avoidance of medical care because of COVID-19-related concerns-United States. Morbidity and Mortality Weekly Report, 69(36), 1250.

Publisher's Note Springer Nature remains neutral with regard to jurisdictional claims in published maps and institutional affiliations. 